\title{
Mesh-morphing algorithms for specimen-specific finite element modeling
}

\author{
Sigal I. A., Hardisty M. R., and Whyne C. M.
}

Version Post-Print/Accepted Manuscript

Citation Sigal IA, Hardisty MR, Whyne CM. Mesh--morphing algorithms for (published version) specimen-specific finite element modeling. J Biomech.

2008;41(7):1381-9. doi: 10.1016/j.jbiomech.2008.02.019. PMID: 18397789

Copyright / License

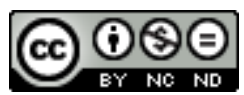

This work is licensed under a Creative Commons Attribution-

NonCommercial-NoDerivatives 4.0 International License.

Publisher's Statement The final published version of this article is available at Elsevier via https://dx.doi.org/10.1016/j.jbiomech.2008.02.019.

How to cite TSpace items

Always cite the published version, so the author(s) will receive recognition through services that track citation counts, e.g. Scopus. If you need to cite the page number of the TSpace version (original manuscript or accepted manuscript) because you cannot access the published version, then cite the TSpace version in addition to the published version using the permanent URI (handle) found on the record page. 


\title{
Mesh-morphing algorithms for specimen-specific finite element
} modeling is

\author{
Ian A. Sigal ${ }^{\mathrm{a}, 1}$, Michael R. Hardisty ${ }^{\mathrm{a}}$, Cari M. Whyne ${ }^{\mathrm{a}, \mathrm{b}, *}$ \\ ${ }^{a}$ Orthopaedic Biomechanics Laboratory, Sunnybrook Health Sciences Centre, 2075 Bayview Avenue, UB19, Toronto, Ontario, Canada M4N 3 M5 \\ ${ }^{\mathrm{b}}$ Institute for Biomaterials and Biomedical Engineering, University of Toronto, Toronto, Ontario, Canada
}

Accepted 21 February 2008

Despite recent advances in software for meshing specimen-specific geometries, considerable effort is still often required to produce and analyze specimen-specific models suitable for biomechanical analysis through finite element modeling. We hypothesize that it is possible to obtain accurate models by adapting a pre-existing geometry to represent a target specimen using morphing techniques. Here we present two algorithms for morphing, automated wrapping (AW) and manual landmarks (ML), and demonstrate their use to prepare specimen-specific models of caudal rat-tail vertebrae. We evaluate the algorithms by measuring the distance between target and morphed geometries and by comparing response to axial loading simulated with finite element (FE).

First a traditional reconstruction process based on $\mu \mathrm{CT}$ was used to obtain two natural specimen-specific FE models. Next, the two morphing algorithms were used to compute mappings from the surface of one model, the source, to the other, the target, and to use this mapping to morph the source mesh to produce a target mesh. The $\mu \mathrm{CT}$ images were then used to assign element-specific material properties. In AW the mappings were obtained by wrapping the source and target surfaces with an auxiliary triangulated surface. In ML, landmarks were manually placed on corresponding locations on the surfaces of both source and target.

Both morphing algorithms were successful in reproducing the shape of the target vertebra with a median distance between natural and morphed models of 18.8 and $32.2 \mu \mathrm{m}$, respectively, for AW and ML. Whereas AW-morphing produced a surface more closely resembling that of the target, ML guaranteed correspondence of the landmark locations between source and target. Morphing preserved the quality of the mesh producing models suitable for FE simulation. Moreover, there were only minor differences between natural and morphed models in predictions of deformation, strain and stress. We therefore conclude that it is possible to use mesh-morphing techniques to produce accurate specimen-specific FE models of caudal rat vertebrae. Mesh morphing techniques provide advantages over conventional specimen-specific finite element modeling by reducing the effort required to generate multiple target specimen models, facilitating intermodel comparisons through correspondence of nodes and maintenance of connectivity, and lends itself to parametric evaluation of "artificial" geometries with a focus on optimizing reconstruction.

(C) 2008 Elsevier Ltd. All rights reserved.

Keywords: Morphing; Finite element modeling; Vertebra; Biomechanics

${ }^{2}$ Supported by the Canadian Institutes of Health Research

*Corresponding author at: Orthopaedic Biomechanics Laboratory, Sunnybrook Health Sciences Centre, 2075 Bayview Avenue, UB19, Toronto, Ontario, Canada M4N 3M5. Tel.: 14164805056 ; fax: 14164805856.

E-mail address: cari.whyne@sunnybrook.ca (C.M. Whyne).

${ }^{1}$ Note: Ian A. Sigal now a post-doctoral research fellow at: the Ocular Biomechanics Laboratory, Devers Eye Institute, Legacy Health Research. Portland, Oregon, USA. isigal@deverseye.org

\section{Introduction}

Detailed finite element (FE) models of biological tissues and structures have been employed successfully and are therefore common in the biomechanics literature. These models are often reconstructed from medical image data, such as computed tomography (CT) or magnetic resonance imaging (MRI), in a process, which draws from an array of segmentation and mesh generation tools to define the geometry. Geometry is then augmented with material constitutive properties, boundary conditions and interac- 


\section{ARTICLE IN PRESS}

\section{Methods}

tions between multi-element models (i.e. soft tissue attachments between bony structures). Even after the model is complete, analysis is complicated by the difficulties in comparing models with varied geometries and differences in discretization (meshing). A traditional approach builds a new model from each new specimen, rendering a specimenspecific model. Work to reduce time and effort required has generally been focused on the amount of manual work and

9 FE expertise necessary through scripts and automating the segmentation (Baghdadi et al., 2005; Barber and Hose, 2005; Grau et al., 2006), geometry reconstruction (Berkley et al., 2000; Couteau et al., 2000; Viceconti et al., 2004; Zhao et al., 2002), mesh generation (Gibson et al., 2003; Liao et al., 2005; Shim et al., 2007; Taddei et al., 2004), material property assignment (Anderson et al., 2005; Taddei et al., 2004, 2007), and analysis (Roberts and Hart, 2005; Sigal et al., 2005). A more efficient method to generate specimen-specific FE models would contribute to analyses dealing with many subjects.

We hypothesize that it is possible to produce accurate specimen-specific model geometries suitable for biomechanical modeling by adapting a pre-existing model using morphing techniques. The objective of this paper is to introduce two algorithms that use information from both source and target geometries to drive the nonlinear deformation process of morphing. We demonstrate the algorithms by using them to prepare specimen-specific models of caudal rat vertebrae. Finally, we evaluate the morphing process by comparing FE-predictions of stress and strain under axial compressive loading between models obtained through morphing (morphed models) and models reconstructed following the standard reconstruction processes (natural models). a

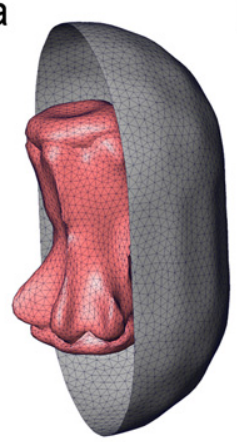

$\operatorname{Energy}(i)=\omega_{1} \sum_{\substack{\text { Tarpet } \\ \text { surface } \\ \text { nodides }}} f(i, j)$
$+\omega_{2} \sum_{\substack{\text { Auxiliany } \\ \text { surfice } \\ \text { neighbors }}} g(i, j)+\omega_{3} h(i)$ b

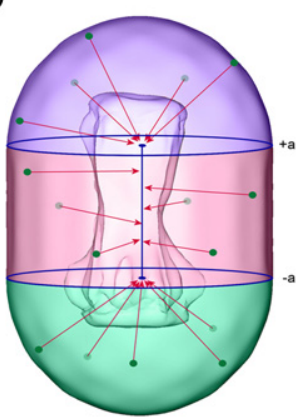

In this paper, we introduce two algorithms to produce subject-specific FE models using morphing. The methods presented herein morph a source model onto the target geometry. Two natural specimen-specific FE models of caudal rat vertebrae were reconstructed from $\mu \mathrm{CT}$ images following a standard iterative process of semi-automated segmentation and surface and tetrahedral volume meshing using Amira (AmiraDev v.3.1, Mercury Computer Systems, USA). The specimens were $\mu \mathrm{CT}$ scanned at a $17.5 \times 17.5 \times 17.5 \mu \mathrm{m} /$ voxel resolution (GE Explore Locus, General Electric Company, Fairfield, USA). Imaging was done with the X-ray source at $90 \mu \mathrm{A}$ and $80 \mathrm{kV}$, with 907 views covering $360^{\circ}$ of rotation. Reconstruction of volumes from the X-ray projections was done with the GE Explore Locus Recon utility to $17.5 \mu \mathrm{m} /$ voxel; the exact image size varied from scan to scan based upon the size and orientation of the specimen. Reconstructions of the source and target vertebrae contained at least 450 slices in the axial direction. Images from the target specimens are then segmented with a simple and quick threshold, to get a rough approximation to the target surface. A mapping is then determined from the surface of the source to the surface of the target. Two algorithms, AW and $\mathrm{ML}$, were used to compute mappings from the surface of the source to that of the target. The algorithms were implemented using $\mathrm{C}++$ (Visual Studio v6.0, Microsoft, USA) and Amira.

\subsection{Automated wrapping ( $A W$ )}

The basic idea of the AW method is that instead of finding a mapping between the complex surfaces of the source and target, it is simpler to find mappings from each of them to an 'auxiliary' surface (Fig. 1). This allowed selecting a convenient shape for the auxiliary surface, which is easy to map onto both source and target. This same technique is used often for morphing in animation (Alexa, 2001; Lazarus and Verroust, 1998; Parus and Kolingerova, 2004; Sheffer and Krayevoy, 2004), or to apply textures to surfaces for graphics processing (Lazarus and Verroust, 1998; Yoshizawa et al., 2004).

The wrapping was done through an energy minimization process. Both the target surface to wrap and the auxiliary surface were considered collections of nodes. The nodes on the target surface were stationary, whereas those on the auxiliary surface started at simple shape and were allowed to displace until a minimum energy configuration was obtained (Fig. 1). The energy function had three components. The first was a longrange energy that drives the initial convergence of the wrapping nodes to

C

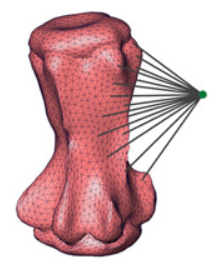

$h(i)=\left\{\begin{array}{l}\alpha \sqrt{x_{i}^{2}+y_{i}^{2}} \text { if }-a<z_{i}<a \\ \alpha \sqrt{x_{i}^{2}+y_{i}^{2}+\left(z_{i}-a\right)^{2}} \text { else }\end{array}\right.$

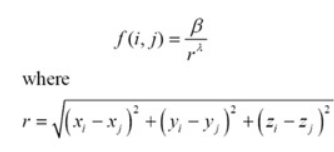

\[ f(i, j)=\frac{\beta}{r^{i}} \]
where
$r=\sqrt{\left(x_{i}-x_{j}\right)^{2}+\left(y_{i}-y_{j}\right)^{2}+\left(z_{i}-z_{j}\right)^{2}}$ d

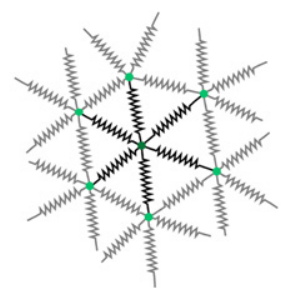

$g(i, j)=V\left(r-r_{0}\right)$

where

$$
\begin{aligned}
& r=\sqrt{\left(x_{i}-x_{j}\right)^{2}+\left(y_{j}-y_{j}\right)^{2}+\left(z_{i}-z_{j}\right)^{2}} \\
& V\left(r-r_{0}\right)=\left\{\begin{array}{l}
\left(r-r_{0}\right)^{n} \text { if }\left(r-r_{0}\right) \geq 0 \\
\left(r-r_{0}\right)^{u} \text { if }\left(r-r_{0}\right) \leq 0
\end{array}\right.
\end{aligned}
$$

Fig. 1. Wrapping energy minimization: An auxiliary surface was wrapped to each of source and target. For clarity only half the auxiliary surface is shown (a). An energy minimization algorithm displaced the auxiliary surface nodes to wrap the target. The energy of a node $i$ on the auxiliary surface had three components: a long-range energy that starts the wrapping so that globally the auxiliary nodes get well distributed over the target surface (b); an energy that drives the detailed convergence of the wrapping to the target surface (c); and an energy that keeps the structure of the auxiliary mesh from distorting badly when converging on the target surface (d). Total nodal energy was minimized using a zero-temperature minimization. 
Wrapped Surface
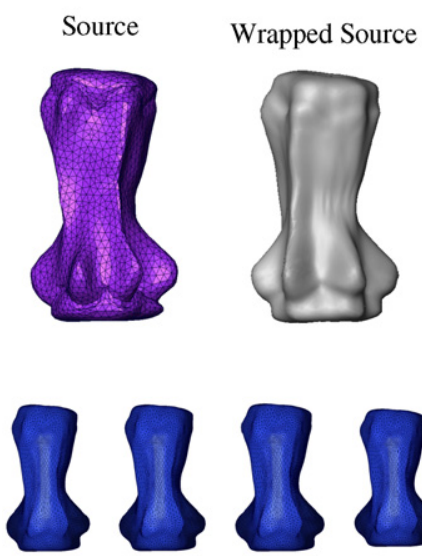

$0 \%$

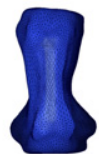

$10 \%$

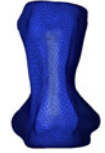

$20 \%$

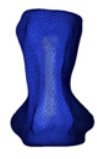

$30 \%$
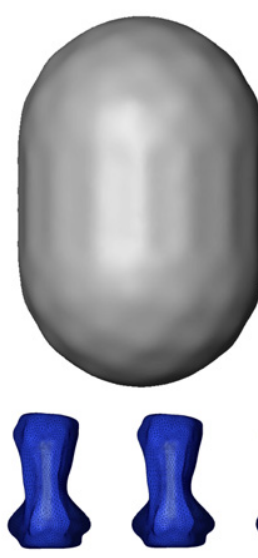

$40 \%$

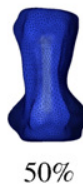

Fig. 2. Automated wrapping: Auxiliary surfaces wrapped to each of source and target. The nodal difference between the two wrapped auxiliary surfaces was then used to compute a transform from the surface of the source to the surface of the target. The source mesh is then morphed onto the target by interpolating the surface transform. The bottom row shows the morphing sequence at various percentage points of the change in geometry. The finite element mesh is valid at every step, but in intermediate steps the material properties are illdefined. At the source $(0 \%)$ and target $(100 \%)$ steps material properties are assigned from the $\mu \mathrm{CT}$ images.

the target. In this way the global distribution of nodes would be relatively homogeneous. The second energy component was a non-linear energy function based on the attractive part of a Lennard-Jones potential (Allen and Tildesley, 1987). This component drives the wrapping to the target surface. The third component of the energy was added to prevent entanglement of the auxiliary surface. This was done by assuming that the nodes of the auxiliary surface were connected with nonlinear springs. This energy had attractive and repulsive parts, with a minimum at the initial distance, i.e. before wrapping starts. The attractive part keeps the overall surface structure unchanged maintaining good nodal distribution. The repulsive part prevents collapse of the auxiliary nodes on the attractive target nodes, and as such has to increase faster for small distances, in this way one node, and only one, of the auxiliary surface can converge onto a node in the surface to wrap.

The energy minimization was implemented through a verlet algorithm much like is done in molecular dynamics when the kinetic energy is kept at zero (Allen and Tildesley, 1987). Following definitions of the target surface and enclosing of the target with the auxiliary wrapping, the attractor regions and parameters of the energy functions are defined. Initial energy for each node on the auxiliary surface is computed and the energy functions are used in an iterative process to minimize the energy state.

Several sets of wrapping parameters were assessed, including various shapes and number of nodes on the auxiliary surface, the power and weight coefficients that determine the energy components, the location and type of the attractors and the energy minimization algorithm. Variations in the parameters produced varying distributions of the nodes once wrapped. Although the wrappings were sensitive to these factors, it was generally easy to get successful wrappings. For the caudal rat vertebrae presented herein a pill-shaped auxiliary surface was found to produce good (smooth with homogeneous node distributions) and repeatable results. Once both source and target were wrapped with the same surface, a mapping between their surfaces could be determined easily from the difference in location of the nodes of the wrapping surface (Fig. 2).

\subsection{Manual landmarks ( $M L)$}

In ML landmarks were manually placed on corresponding locations on the surfaces of both source and target vertebrae. Eleven equally spaced planes orthogonal to the main axis of the vertebra were defined. Twelve landmarks were placed on each of the central nine planes (total of 108) (Fig. 3). Landmarks were placed on the tips of the processes and valleys between processes, which were always easy to determine. Using outlines of the vertebra on the plane landmarks were forced to be on the vertebral surface. A mapping was then produced to match the target and source landmarks. Thin-plate splines were used which produce a nice smooth transformation and allow precise transformation of point $\mathrm{A}$ to $\mathrm{A}^{\prime}$. We used the classical Bookstein thin-plate splines transformation as implemented in Amira (Bookstein, 1987; Zelditch et al., 2004).

When a candidate mapping between source and target surfaces has been computed, either by AW or ML, it can be tested by morphing only the surface of the source model. This provides a preview of what the full mesh morphing produces. The mappings can then be adjusted or redone. Once a satisfactory mapping has been found the full 3D mesh of the source model is morphed onto the target. This can be done through interpolation from the surfaces (both linear and cubic interpolations were used), or through thin-plate splines using all source and target surface nodes as landmarks.

\subsection{Simulation}

Elements were assigned Young's modulus based on their mean intensity in the $\mu \mathrm{CT}$ images stack using Bonemat (Taddei et al., 2004, 2007), and a constant Poisson ratio of 0.3 (Chevalier et al., 2007). Material properties were assumed linearly elastic and transversely isotropic, with an elastic modulus three times higher in the axial direction than in the plane orthogonal to the axis (Taddei et al., 2004, 2007; Wilcox, 2007).

We present here four models, two natural models from two rat caudal vertebral specimens, and two models obtained by morphing one source vertebra to the other target vertebra using the AW and the ML methods. Each of these models was used to simulate the mechanical response of the vertebra to axial compressive loading (Abaqus, version 6.5.1 Dassault Systems, Providence, RI, USA). A force of $25 \mathrm{~N}$ was applied to the caudal surface while nodes on the rostral surface were prevented from displacing in the rostral-caudal direction (but not in the dorsal-ventral or lateral directions).

Adequate mean element size was determined in a preliminary mesh refinement study carried out on one of the natural models based on the median von Mises stress and the median axial strain. Since optimal element size could potentially depend on the model used in the refinement the optimal mean element size was reduced by $30 \%$. This also allowed for sufficient resolution when models from small specimens were morphed to larger specimens, increasing mean element size (decreasing element density). 


\section{ARTICLE IN PRESS}

Fig. 3. Manual landmarks: Landmarks were placed manually on source (left) and target (right). A thin-plate splines method was then used to compute the full-field transformations between source and target. The method guarantees coincidence between corresponding landmarks. The middle column shows landmarks on the source (gray) and target (yellow) geometries on a semi-transparent target geometry. Corresponding landmarks are connected with a red line. Clearly visible on the top and bottom row of landmarks are mostly vertical lines representing the differences in height between source and target.

Table 1

Comparison of geometric fidelity, mesh quality and predicted response to loading of two natural and two morphed models
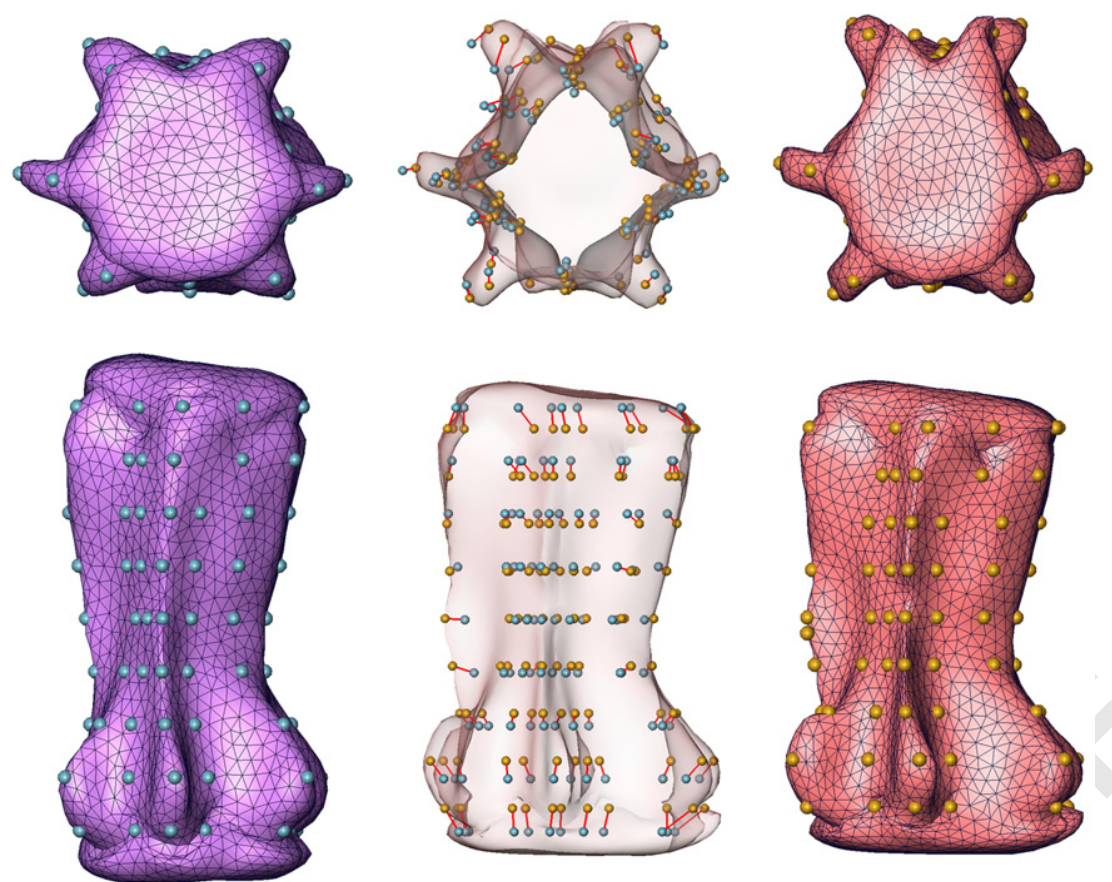

\begin{tabular}{llllll}
$\begin{array}{l}\text { Median distance } \\
\text { to target natural } \\
\text { surface }(\mu \mathrm{m})\end{array}$ & $\begin{array}{l}\text { Distorted } \\
\text { elements }\end{array}$ & $\begin{array}{l}\text { Normalized } \\
\text { median axial } \\
\text { strain }\end{array}$ & $\begin{array}{l}\text { Normalized } \\
\text { median von } \\
\text { mises stress }\end{array}$ & $\begin{array}{l}\text { Normalized peak } \\
\text { axial strain }\end{array}$ & $\begin{array}{l}\text { Normalized peak } \\
\text { von mises stress }\end{array}$ \\
\hline- & $0 / 36769$ & 1.00 & 1.00 & 1.00 & 1.00 \\
18.8 & $28 / 54798$ & 0.9889 & 0.9483 & 0.9795 & 0.9303 \\
32.2 & $9 / 54798$ & 0.9619 & 0.9742 & 0.9538 & 0.9453 \\
$>350$ & $10 / 54798$ & 0.8696 & 0.7075 & 0.7848 & 0.7520 \\
\hline
\end{tabular}

Median and peak (50th and 95th percentiles, respectively) strain and stress are normalized to that of the natural model of the target to simplify comparison. The measures for the natural models of the source were included to provide an idea of the typical variability in response between specimens, since morphing would provide no substantial contribution if predictions in all specimens were essentially the same.

We evaluated the morphings by the fidelity of the geometry, the quality of the mesh and the differences in response to loading predicted with FE between morphed and natural models. Fidelity of the morphed geometry was determined by comparing the distance between surfaces of the natural and morphed models. For the quality of the mesh we used the internal routines of Abaqus to determine the number of distorted elements before and after morphing. This routine uses a combination of element size, aspect ratio and the angle between faces to judge element quality (Abaqus, 2006; Couteau et al., 2000). Differences in response to loading were measured comparing median (50th percentile) and peak (95th percentile) axial strain and Von Mises stress.

\section{Results}

Both morphing algorithms were successful in producing models of the target vertebra suitable for FE simulation while preserving the quality of the mesh (Table 1). Fig. 4 shows an example of typical results of morphing a geometry. Slight differences in the geometry can be appreciated. AW seems to have produced geometry closer to the natural target. However, models morphed through AW do not guarantee that there is point to point correspondence, only that the shapes are the same. Models morphed through ML have correspondence at the landmarks, but regions between landmarks can differ. AW has difficulty in wrapping small and relatively convoluted regions, so that in these regions the shapes do not agree and might in fact be slightly distorted in a model morphed through AW (see for example the rostral growth plate region on the bottom right of the $\mathrm{AW}$-morphed model in Fig. 4). ML did not produce a distorted model, but in 


\section{ARTICLE IN PRESS}
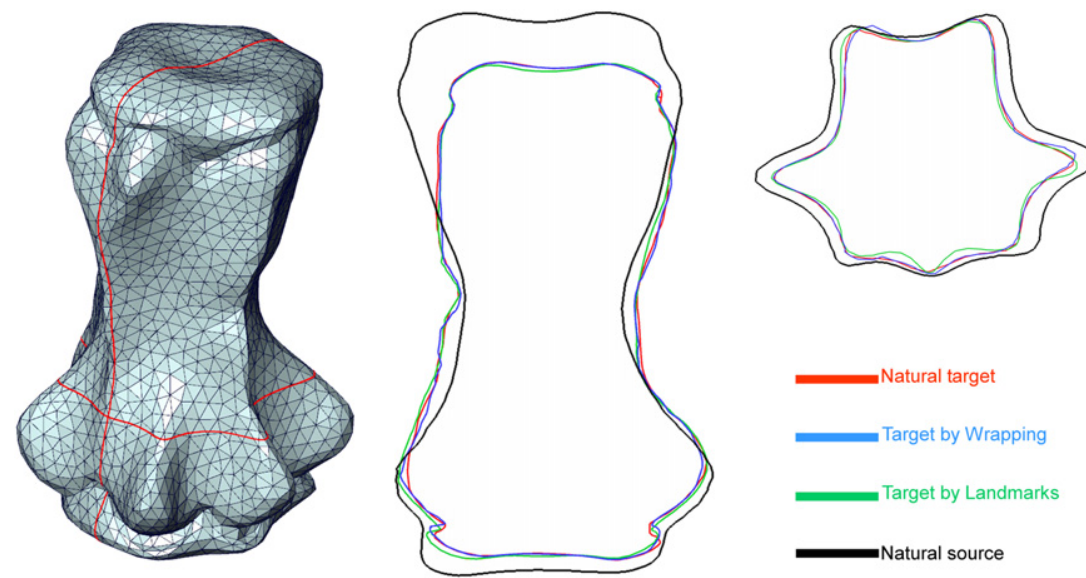

Fig. 4. Geometry correspondence and morphing: Surface of the target model (top left). Comparison of coronal (top center) and sagittal (top right) cross sections of the target model (red line), AW (blue line) and ML (green). Median distance to the natural surface is $18.8 \mu \mathrm{m}$ for AW, and $32.2 \mu \mathrm{m}$ for ML and $350 \mu \mathrm{m}$ for source. Length of the vertebra is approximately $7000 \mu \mathrm{m}$.
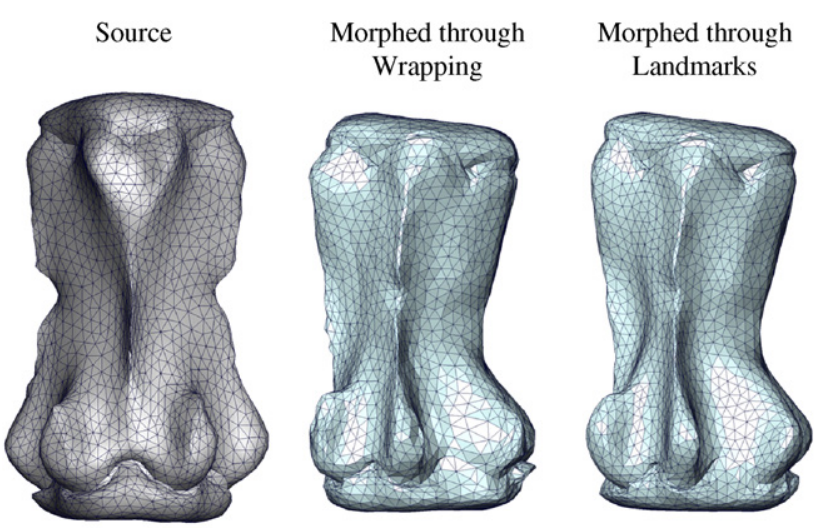

$$
\text { Target }
$$
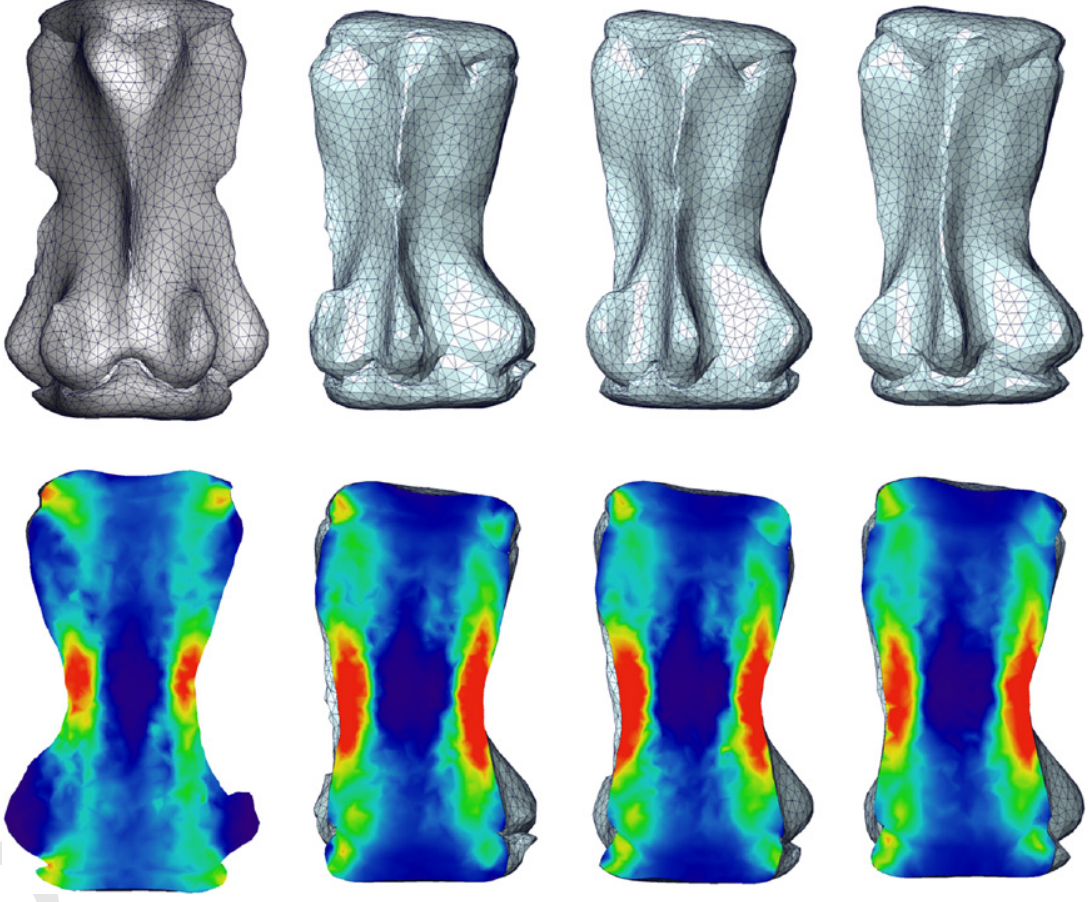

Fig. 5. Exterior views (top row) and cross sections (bottom row) through the source (left column) and target (right column) models, as well as two versions of the source morphed onto the target through wrapping (second column from the left) and landmarks (third column from the left). The cross sections are colored according to the magnitude of von Mises equivalent stress predicted using FEM for a $25 \mathrm{~N}$ load on the caudal side (top). The rostral side (bottom) was forced to remain stationary. Transversely isotropic material properties were assigned based on the $\mu \mathrm{CT}$-intensity, with elastic modulus three times higher in the axial direction (vertical in the images above) than in the plane orthogonal to this axis. A comparison of the median and peak von Mises stress between the models is presented in Table 1. Stress magnitudes were normalized to the peak (95th percentile) of the source model.

regions distant from landmarks the agreement with the target could potentially be poor. By better matching the surfaces the AW allowed for models that captured better the cortical bone, and therefore was able to approximate better the median stresses predicted with the natural model (Fig. 5). When correspondence between locations is important, as in models with complex boundary condi- tions, like ligamentous attachments, some level of correspondence between locations by using ML may be required. Morphed models always more closely reproduced the median than the peak responses, and in no case overpredicted the levels of strain or stress.

The algorithms presented here not only satisfied the demand of producing smooth transformations in space 
1 that do not distort a mesh to the point of compromising its usability for FEA, in some cases the quality of the mesh was slightly improved (see Table 1). In all models the number of distorted elements was very small, less than $0.05 \%$ of the elements. This proportion is on the same order as what has been observed with model morphing using other algorithms (Bade et al., 2006; Krause and Sander, 2006; Lee et al., 2006; Shontz and Vavasis, 2003).

\section{Discussion and conclusions}

Mesh-morphing techniques have allowed production of specimen-specific FE models of caudal rat vertebrae using an independent source mesh. Predictions of deformation, strain and stress of the target vertebra under axial loading have only minor differences between morphed and individually generated models. These small differences may depend on the particular loading configuration studied, an aspect which we are currently studying through a sensitivity analysis on geometry, material properties and loadings to determine the relative influence of each factor on the mechanical response.

The morphing methods described here are aimed at improving the overall efficiency of specimen-specific analysis, not at simply making the step of meshing bony structures faster. Fast specimen-specific meshing programs already exist to model bone imaged with CT scans (Viceconti et al., 2004). Our intention was to use a single source model to simplify some steps of pre-processingand post-processing, such as applying boundary conditions and direct comparison of simulation outputs between models. The methods we present do, however, impose an overhead on other aspects of pre-processing, namely the identification of the auxiliary surface, of a useful set of landmarks and adjustments of the mappings. However, once these have been identified for a given structure type, it is generally fast to apply them to new specimens. For example, with these issues now resolved for rat caudal vertebrae, generating models is actually faster than with traditional specimen-specific FE meshing techniques. The model generation still requires the placement of landmarks or wrapping of the target geometry, but at known locations and with known parameters.

The ability to generate fast meshes for bone is not necessarily sufficient for modeling of the spinal motion segments, which include discs, ligaments and articulating surfaces. Thus, the techniques we have presented are aimed at creating an advantage over meshing of more multifaceted structures. Attachments to the vertebral body such as the intervertebral disc and liagmentous structures, or loading conditions, can be maintained through the morphing process more easily in ML-morphed models, significantly simplifying the development of specimenspecific FE spinal motion segment models. As such, the complexity of the lamellar structure of the annulus fibrosus attached to the endplate of the vertebral body developed for a single-source model could be utilized repeatedly in developing multiple specimen-specific models without the need to redefine attachment points and orientations. Such complexities are not included in our demonstration case within this manuscript but would represent a next step following the proof of this process.

Utilizing models that share meshes yields benefits apparent during post-processing, where routines and techniques to compare outcomes are simplified thanks to the correspondence of meshes, i.e. nodes, connectivity and overall topology, between models Beyond pre- and postprocessing morphing methods allow other opportunities. For example, a morphing mapping between specific geometries can produce a field useful to assess differences in morphology, similar to the field analysis used in morphometric studies of the evolution of shapes (Zelditch et al., 2004; Richtsmeier et al., 2002). Morphing also allows the generation of a series of models at several steps between two (or more) geometries, to study the full geometry factor space between them. With respect to analyzing vertebrae, morphing may be used to change single factors such as the vertebral scale or the shape of the processes, producing "artificial" geometries that are derived from actual shapes. As such, morphing provides an ideal platform for conducting parametric evaluation of the sensitivity to geometry.

Herein we focus on model geometry because methods to assign material properties based on $\mu \mathrm{CT}$ images are reasonably well automated for our work. The mesh morphing can easily be generalized to several wrapped surfaces, internal landmarks, or a combination of both. We have already tapped into this possibility by using a combination of AW and ML to deform models of the eye based on $\mu$ MRI (Sigal et al., 2007). Although in general it would be preferable to use higher order and/or hexahedral elements for FE modeling (Cook, 1995; Lee et al., 2006), for the proof-of-concept purposes of this work it is believed that linear tetrahedral elements suffice. In theory there is nothing that prevents applying our morphing techniques to higher order elements or hexahedrals. Furthermore, we have applied the morphing methods to several other specimens, including the same pair of natural models but with source and target roles inverted with similar results.

As would be expected, the methods do have some limitations that deserve consideration. Use of smooth transformations between the source and target allow morphings that do not distort the FE mesh, but any inherent problems or limitations, which exist in the original source mesh would be propogated. The methods require the surfaces of both the source and target with the two morphing methods differing in the amount and type of manual work required by an experienced analyst to guide the morphing process. While relatively detailed source and tatget surfaces were utilized during the development phase of the algorithms, substantial improvements both in reducing user effort and increasing in robustness to imaging limitations and artefacts are possible. In land- 
1 marks-based morphing improvements would come by identifying a minimal set of landmarks. Preliminary results suggest that the number of landmarks can be substantially reduced without sacrificing much in terms of performance. Reductions in user effort could also be realized through automating landmark placement, although automatically defining unequivocal landmarks in biological objects is not trivial and often difficult to generalize (Berkley et al., 2000; Bookstein, 1987; Bowden et al., 1998b; Lapeer and Prager, 2000; Lazarus and Verroust, 1998; Richtsmeier et al., 2002; Yoshizawa et al., 2004). We found identifying landmarks visually simple, and therefore minimizing the number of landmarks was not one of our objectives. Variations in landmark locations due to small errors or noise would lead to slightly different morphed shapes, but likely not to substantially different mechanical responses. The same would be the case for variations on vertebral morphed shape due to changes in auxiliary surface or morphing functions. This was tested by replicating the landmarkbased morphing several weeks after the first definitions. The mechanical responses of the two landmark-based morphings were virtually indistinguishable.

In wrapping-based morphing improvements would come from identifying wrapping surfaces that are simpler yet allow tighter and more homogenous wrapping for a wider variety of geometries, perhaps using a vertebral atlas, as we have done for image segmentation (Hardisty et al., 2007). The choice of an auxiliary surface together with a wrapping algorithm influences the performance of AW techniques. The goal of the auxiliary surface and wrapping algorithm were to provide an accurate and smooth mapping between the surfaces of the wrapped objects. A good mapping has the nodes on the wrapping distributed relatively homogeneously, but with all the relevant structures properly represented (i.e. wrapped by enough nodes on the right places). Naturally, it is not always possible to know a priori which are the relevant structures and the detail with which they have to be represented, as this may depend on other conditions (i.e. loading). A common way to determine the relevance of different structures is through a geometric sensitivity study. Hybrid methods in the form of a landmark-guided wrapping could potentially bring together benefits of each of the techniques, for example using a small set of landmarks as attractors to guide the initial phase of the wrapping, as we have done in modeling eye geometries (Sigal et al., 2007). However, eye geometries are close to spheres and therefore wrapping methods more robust for this application; the exact form of a hybrid method useful in orthopaedic applications such as the vertebrae requires further investigation. In this initial work, rodent caudal vertebrae were used which are relatively similar from a geometric perspective. Future studies will extend these techniques to more dissimilar vertebrae further challenging the algorithms.

Semi-automatic and automatic template-based warping techniques have been applied to solve a broad spectrum of problems in mechanics (Lamecker et al., 2004; Zockler et al., 2000), nonlinear strain computation (Bowden et al., 1997; Phatak et al., 2007; Veress et al., 2002), and medical image segmentation (Bowden et al., 1998a,b). The present work applied the same technique to generate subjectspecific FE models on the basis of warping template models. The idea of morphing has also been used to make up for sparsity or low quality in the target dataset (Blanz et al., 2004; Lapeer and Prager, 2000; Shim et al., 2007). Others have done morphing based on template finite element models whereby the elements of the source model are assigned material properties and morphed onto the target through application of FE. In this technique nonlinear continuum mechanics are used to guarantee a continuous mapping between source and target. This has the possible negative consequence that it could potentially overpenalize some deformation fields, generally requiring ad hoc manual selection of material properties. Such mechanics-based methods have more demanding computational requirements in comparison to the simpler morphing methods presented here (Brock et al., 2005a, b).

Within the animation and computer graphics community, much morphing has been carried out based on the ideas of free form deformation (FFD) initially developed by Sederberg and Parry (1986). In FFD both source and target geometries are embedded in a control volume, which is deformed using a set of landmarks as guide, passing the deformation to the embedded objects. More recently, FFD-based techniques have been applied to the study of biomechanics, and other organs, but to the best of our knowledge not to vertebral biomechanics (Fernandez et al., 2005, 2004). Despite their successful application, FFD techniques share some limitations with the methods presented in this work. Most notably that they still require a considerable amount of manual work, and some trial and error to determine adequate control volumes and landmarks to guide the deformation (Fernandez et al., 2004). FFD techniques, like AW, require the source and target geometries to be similar. Several of the implementations of FFD have been optimized for animation, producing smooth transformation fields and requiring a smaller set of landmarks than ML, but cannot guarantee correspondence between source and target landmarks as ML. Despite these limitations FFD methods are a good alternative for morphing for sensitivity studies.

The methods presented here have several other advantages over conventional specimen-specific finite element modeling: (1) Once the reference FE model is generated in the conventional manner, less effort is required to generate models of the target specimen. This is potentially critical for multi-element models, where the interactions between the elements are especially difficult or time consuming to define. (2) Models that share the mesh are easier to compare due to the correspondence of nodes and connectivity facilitating the use of scripts for automated pre and post-processing. (3) Morphing can be extended to allow parametric evaluation of "artificial" or non-existent geometries. 
In conclusion, the methods presented here have several advantages over conventional specimen-specific FE modeling: (1) Once the reference FE model is generated in the conventional manner, less effort is required to generate models of the target specimen. This is potentially critical for multi-element models, where the interactions between the elements are especially difficult or time consuming to define. (2) Models that share the mesh are easier to compare due to the correspondence of nodes and connectivity facilitating the use of scripts for automated pre and post-processing. (3) Morphing can be extended to allow parametric evaluation of "artificial" or non-existent geometries. This paper has introduced morphing methods to produce 3D specimen-specific models of rat-tail vertebrae for FE analysis. The originality of this work lies in the application of morphing techniques to help produce and simplify analysis of FE models. While neither the source model generation the morphing nor FE-analysis routines are novel, the integration of these techniques shows a new method for the comparative mechanical analysis of multiple vertebrae and ultimately spinal motion segments.

\section{Conflict of interest statement}

\section{None.}

\section{Acknowledgments}

The authors are grateful to Tomasz D. Szwedowski for the valuable discussions, and acknowledge the financial support of the Canadian Institutes of Health Research.

\section{References}

Abaqus, 2006. Abaqus v6.5.1 Online Reference Manual. Dassault Systems, Providence, RI, USA.

Alexa, M., 2001. Local control for mesh morphing. In: International Conference on Shape Modeling and Applications, IEEE Computer Society.

Allen, M.P., Tildesley, D.J., 1987. Computer Simulation of Liquids. Clarendon Press, Oxford.

Anderson, A.E., Peters, C.L., Tuttle, B.D., Weiss, J.A., 2005. Subjectspecific finite element model of the pelvis: development, validation and sensitivity studies. Journal of Biomechanical. Engineering. 127, 364-373.

Bade, R., Haase, J., Preim, B., 2006. Comparison of Fundamental Mesh Smoothing Algorithms for Medical Surface Models, Simulation und Visualisierung 2006 (SimVis 2006). SCS Publishing House e.V., Magdeburg, pp. 289-304.

Baghdadi, L., Steinman, D.A., Ladak, H.M., 2005. Template-based finiteelement mesh generation from medical images. Computer Methods and Programs in Biomedicine 77, 11-21.

Barber, D.C., Hose, D.R., 2005. Automatic segmentation of medical images using image registration:diagnostic and simulation applications. Journal of Medical Engineering and Technology 29, 53-63.

Berkley, J., Oppenheimer, P., Weghorst, S., Berg, D., Raugi, G., Haynor, D., Ganter, M., Brooking, C., Turkiyyah, G., 2000. Creating fast finite element models from medical images. Studies in Health Technology and Informatics 70, 26-32.

Blanz, V., Mehl, A., Vetter, T., Seidel, H.-P., 2004. A Statistical Method for Robust 3D Surface Reconstruction from Sparse Data. In: 3D Data
Processing, Visualization, and Transmission, Secondnd International Symposium on (3DPVT'04), IEEE Computer Society, pp. 293-300.

Bookstein, F.L., 1987. Describing a craniofacial anomaly: finite elements and the biometrics of landmark locations. American Journal of Physical Anthropology 74, 495-509.

Bowden, A.E., Rabbitt, R.D., Weiss, J.A., 1998a.Warping template finite element models into alignment with subject specific image data. ASME Winter Annual Meeting. Annaheim, CA.

Bowden, A.E., Rabbitt, R.D., Weiss, J.A., 1998b. Anatomical registration and segmentation by warping template finite element models. In: Jacques, S. L. (Ed.), Society of Photo-Optical Instrumentation Engineers (SPIE) Conference, pp. 469-476.

Bowden, A.E., Rabbitt, R.D., Weiss, J.A., Maker, B.N., 1997. Use of medical image data to compute strain fields in biological tissue. American Society of Mechanical Engineering BED, 191-192.

Brock, K.K., Dawson, L.A., Sharpe, M.B., Moseley, D.J., Jaffray, D.A., 2005. Feasibility of a novel deformable image registration technique to facilitate classification, targeting, and monitoring of tumor and normal tissue. International Journal of Radiation Oncology, Biology and Physics 64, 1245-1254.

Brock, K.K., Sharpe, M.B., Dawson, L.A., Kim, S.M., Jaffray, D.A., 2005. Accuracy of finite element model-based multi-organ deformable image registration. Medical Physics 32, 1647-1659.

Chevalier, Y., Pahr, D., Allmer, H., Charlebois, M., Zysset, P., 2007. Validation of a voxel-based FE method for prediction of the uniaxial apparent modulus of human trabecular bone using macroscopic mechanical tests and nanoindentation. Journal of Biomechanics.

Cook, R.D., 1995. Finite Element Modeling for Stress Analysis. Wiley, New York.

Couteau, B., Payan, Y., Lavallee, S., 2000. The mesh-matching algorithm: an automatic 3D mesh generator for finite element structures. Journal of Biomechanics 33, 1005-1009.

Fernandez, J.W., Mithraratne, P., Thrupp, S.F., Tawhai, M.H., Hunter, P.J., 2004. Anatomically based geometric modelling of the musculoskeletal system and other organs. Biomechanics Model and Mechanobiology 2, 139-155.

Fernandez, J.W., Ho, A., Walt, S., Anderson, I.A., Hunter, P.J., 2005. A cerebral palsy assessment tool using anatomically based geometries and free-form deformation. Biomechanics Model and Mechanobiology 4, 39-56.

Gibson, A.P., Riley, J., Schweiger, M., Hebden, J.C., Arridge, S.R., Delpy, D.T., 2003. A method for generating patient-specific finite element meshes for head modelling. Physics in Medicine and Biology $48,481-495$.

Grau, V., Downs, J.C., Burgoyne, C.F., 2006. Segmentation of trabeculated structures using an anisotropic Markov random field: application to the study of the optic nerve head in glaucoma. IEEE Transactions on Medical Imaging 25, 245-255.

Hardisty, M., Gordon, L., Agarwal, P., Skrinskas, T., Whyne, C., 2007. Quantitative characterization of metastatic disease in the spine. Part I. Semiautomated segmentation using atlas-based deformable registration and the level set method. Medical Physics 34, 3127-3134.

Krause, R., Sander, O., 2006. Automatic construction of boundary parametrizations for geometric multigrid solvers. Computer Vision and Science 9, 11-22.

Lamecker, H., Seebass, M., Lange, T., Hege, H.C., Deuflhard, P., 2004. Visualization of the variability of $3 \mathrm{D}$ statistical shape models by animation. Studies in Health Technology and Information 98, 190-196.

Lapeer, R.J., Prager, R.W., 2000. 3D shape recovery of a newborn skull using thin-plate splines. Computerized Medical Imaging and Graphics 24, 193-204.

Lazarus, F., Verroust, A., 1998. Three-dimensional metamorphosis: a survey. The Visual Computer 14, 373-389.

Lee, C.F., Chen, P.R., Lee, W.J., Chen, J.H., Liu, T.C., 2006. Threedimensional reconstruction and modeling of middle ear biomechanics by high-resolution computed tomography and finite element analysis. Laryngoscope 116, 711-716. 
Liao, S., Tong, R., Wang, M., Dong, J., 2005. Rapidly generate lumbar spine volume mesh. In: Ninth International Conference on Computer Aided Design and Computer Graphics, 2005. IEEE Computer Society, pp. 345-350.

Parus, J., Kolingerova, I., 2004. Morphing of meshes with attributes. In: 20th Spring Conference on Computer Graphics, Budmerice, Slovakia, pp. $73-81$.

Phatak, N.S., Sun, Q., Kim, S.E., Parker, D.L., Kent Sanders, R., Veress, A.I., Ellis, B.J., Weiss, J.A., 2007. Noninvasive determination of ligament strain with deformable image registration. Annals of Biomedical Engineering 35, 1175-1187.

Richtsmeier, J.T., DeLeon, V.B., Lele, S.R., 2002. The promise of geometric morphometrics. American Journal of Physical Anthropology Supplment 35, 63-91.

Roberts, M.D., Hart, R.T., 2005. Shape adaptation of long bone structures using a contour based approach. Computer Methods in Biomechanics and Biomedical Engineering 8, 145-156.

Sederberg, T.W., Parry, S.R., 1986. Free-form deformation of solid geometric model. In: Evans, D.C., Athay, R.J. (Eds.), SIGGRAPH 86, ACM SIGGRAPH. ACM, New York, pp. 151-160.

Sheffer, A., Krayevoy, V., 2004. Shape preserving mesh deformation. In: International Conference on Computer Graphics and Interactive Techniques. SIGGRAPH, Los Angeles, CA, p. 39.

Shim, V.B., Pitto, R.P., Streicher, R.M., Hunter, P.J., Anderson, I.A., 2007. The use of sparse CT datasets for auto-generating accurate FE models of the femur and pelvis. Journal of Biomechanics 40, 26-35.

Shontz, S.M., Vavasis, S.A., 2003. A mesh warping algorithm based on weighted Laplacian smoothing. In: 12th International Meshing Roundtable, Santa Fe, NM, pp. 147-158.

Sigal, I.A., Flanagan, J.G., Ethier, C.R., 2005. Factors influencing optic nerve head biomechanics. Investigative Ophthalmology and Visual Science 46, 4189-4199.

Sigal, I.A., Norman, R.E., Rausch, S.M.K., Tertinegg, I., Eilaghi, A., Morgan, K., Portnoy, S., Sled, J.G., Flanagan, J.G., Ethier, C.R.,
2007. Mechanics of individual-specific models of the corneo-scleral shell in glaucoma, invest. Ophthalmology and Visual Science 48 (EAbstract 3305).

Taddei, F., Pancanti, A., Viceconti, M., 2004. An improved method for the automatic mapping of computed tomography numbers onto finite element models. Medical Engineering and Physics 26, 61-69.

Taddei, F., Schileo, E., Helgason, B., Cristofolini, L., Viceconti, M., 2007. The material mapping strategy influences the accuracy of CT-based finite element models of bones: an evaluation against experimental measurements. Medical Engineering and Physics 29, 973-979.

Veress, A.I., Weiss, J.A., Gullberg, G.T., Vince, D.G., Rabbitt, R.D., 2002. Strain measurement in coronary arteries using intravascular ultrasound and deformable images. Journal of Biomechical Engineering 124, 734-741.

Viceconti, M., Davinelli, M., Taddei, F., Cappello, A., 2004. Automatic generation of accurate subject-specific bone finite element models to be used in clinical studies. Journal of Biomechanics 37, 1597-1605.

Wilcox, R.K., 2007. The influence of material property and morphological parameters on specimen-specific finite element models of porcine vertebral bodies. Journal of Biomechanics 40, 669-673.

Yoshizawa, S., Belyaev, A.G., Seidel, H.-P., 2004. A fast and simple stretch-minimizing mesh parameterization. In: International Conference on Shape Modeling and Applications, Genova, Italy, pp. 200-208.

Zelditch, M., Swiderski, D., Sheets, D., Fink, W., 2004. Geometric Morphometrics for Biologists: a Primer. Elsevier Academic Press, London.

Zhao, L., Han, H., Patel, P.K., Widera, G.E., Harris, G.F., 2002. Finite element (FE) modeling of the mandible: from geometric model to tetrahedral volumetric mesh. Studies in Health Technology and Information 85, 593-596.

Zockler, M., Stalling, D., Hege, H.-C., 2000. Fast and intuitive generation of geometric shape transitions. The Visual Computer 16, 241-253. 